\title{
Kelley's specialization of Tychonoff's Theorem is equivalent to the Boolean Prime Ideal Theorem
}

\author{
by
}

\author{
Eric Schechter (Nashville, TN)
}

\begin{abstract}
The principle that "any product of cofinite topologies is compact" is equivalent (without appealing to the Axiom of Choice) to the Boolean Prime Ideal Theorem.
\end{abstract}

1. Introduction. The principle that is nowadays commonly known $\left({ }^{1}\right)$ as Tychonoff's Theorem states that

(TT) any product of compact spaces is compact,

when the product space is equipped with the product topology. It was proved in 1930s by several methods, all using the Axiom of Choice $\left({ }^{2}\right)$ (AC). In 1950 John L. Kelley published a proof of the converse, TT $\Rightarrow$ AC, thus demonstrating equivalence of the two principles. His proof contained a very minor error $\left({ }^{3}\right)$, which is easily corrected. This was mentioned by Łoś and Ryll-Nardzewski in 1951; a corrected proof was published by Plastria in 1972. Incidentally, Plastria's proof also shows that TT and AC are equivalent to

2000 Mathematics Subject Classification: Primary 03E25.

Key words and phrases: Axiom of Choice, Boolean Prime Ideal Theorem, generalized sequence, Moore-Smith sequence, net, product topology, Tychonoff's Theorem, ultrafilter, universal net.

( $\left.{ }^{1}\right)$ Actually, what Tychonoff himself proved is the more specialized result $\mathrm{TT}_{I}$, listed later in this section. The formulation that we are calling TT was given later by Čech.

$\left({ }^{2}\right)$ The Axiom of Choice, in its simplest form, says that any product of nonempty sets is nonempty; we may arbitrarily choose a member from each of those nonempty sets. For the benefit of any newcomers to this subject, we restate the axiom in other terms: AC is a nonconstructive assertion of existence, requiring a formalist philosophy of mathematics. When we accept AC, we are agreeing to the convention that, even if we are unable to exhibit a particular example of a member of a product of nonempty sets, we are still permitted to use a hypothetical member of that product in proofs, as though it exists in some sense.

$\left({ }^{3}\right)$ Unfortunately, Kelley's error was propagated in my book [9]. I am grateful to Michael Greinecker for bringing it to my attention. 
the statement that any product of compact $T_{1}$ spaces is compact; see related remarks at the end of this section.

Kelley had argued $\mathrm{TT} \Rightarrow \mathrm{TT}_{\mathrm{cf}} \stackrel{*}{\Rightarrow} \mathrm{AC}$, using the intermediate principle

$\left(\mathrm{TT}_{\mathrm{cf}}\right)$ any product of cofinite topologies is compact,

but his proof of $\stackrel{*}{\Rightarrow}$ was faulty. Plastria's corrected proof of $\mathrm{TT} \Rightarrow \mathrm{AC}$ did not involve $\mathrm{TT}_{\text {cf }}$, and left open this question: Is the implication $\stackrel{*}{\Rightarrow}$ true but unproved, or is it actually false?

In this note we shall show that $\stackrel{*}{\Rightarrow}$ is false. It turns out that $\mathrm{TT}_{\mathrm{cf}}$ is equivalent to the Boolean Prime Ideal Theorem (BPI), a principle well known $\left({ }^{4}\right)$ to be strictly weaker than AC.

This note is not actually concerned with Boolean prime ideals. We have mentioned BPI only as an identifier; it is the most famous of a whole family of principles known to be equivalent to one another. Here are four members of that family:

$\left(\mathrm{TT}_{2}\right) 2^{J}$ is compact for any set $J$, if $2=\{0,1\}$ has the discrete topology.

$\left(\mathrm{TT}_{I}\right)[0,1]^{J}$ is compact, for any set $J$.

$\left(\mathrm{TT}_{\mathrm{h}}\right)$ Any product of compact Hausdorff spaces is compact.

(U) A topological space $P$ is compact if and only if every universal net in $P$ converges to at least one limit in $P$.

Obviously Kelley's principle $\mathrm{TT}_{\mathrm{cf}}$ implies Mycielski's principle $\mathrm{TT}_{2}$. To establish equivalence, we shall show that the universal net principle $\mathrm{U}$ implies $\mathrm{TT}_{\mathrm{cf}}$.

$\mathrm{TT}_{\mathrm{h}}$ and $\mathrm{TT}_{2}$ have often been useful in the study of equivalents of BPI, because a number of compactness principles $\mathrm{C}$ are trivially seen to satisfy $\mathrm{TT}_{\mathrm{h}} \Rightarrow \mathrm{C} \Rightarrow \mathrm{TT}_{2}$. However, Kelley's principle $\mathrm{TT}_{\mathrm{cf}}$ does not yield to that analysis; the cofinite topology on any infinite set is $\mathrm{T}_{1}$ but not Hausdorff.

2. Tutorial on nets. Some readers may be unfamiliar with nets and with universal nets; to make this paper self-contained, we now give a brief tutorial on that subject. A more detailed introduction can be found in [5] or [9].

Sequences $\left(x_{n}: n \in \mathbb{N}\right)$ are useful tools in metric spaces and in some other topological spaces. For analogous tools in arbitrary topological spaces one may turn to nets (also known as generalized sequences or as MooreSmith sequences). These may be written in the form $\left(x_{\delta}: \delta \in \mathbb{D}\right)$, where the subscripts $\delta$ are members of any directed set-i.e., a set $\mathbb{D}$ whose ordering $\preccurlyeq$ is reflexive and transitive and has the further property that each finite subset of $\mathbb{D}$ has a $\preccurlyeq$-upper bound in $\mathbb{D}$.

$\left({ }^{4}\right)$ Proved by Halpern and Lévy [2]. See [3], [9], and sources cited therein for further discussion of AC, BPI, and their relatives. 
A net $\left(x_{\delta}\right)$ is said to satisfy some condition eventually if the condition is satisfied by $x_{\delta}$ for all $\delta$ later than some $\delta_{0}$. A net $\left(x_{\delta}\right)$ is universal if for each set $S$ we have either eventually $x_{\delta} \in S$ or eventually $x_{\delta} \notin S$. For example, if a net is eventually constant, then it is universal $\left({ }^{5}\right)$. Conversely, if a universal net takes values in a finite set, then the net must be eventually constant.

In a topological space, we say that a net $\left(x_{\delta}\right)$ is convergent to a limit $z$ (written $x_{\delta} \rightarrow z$ ) if $x_{\delta}$ is eventually in each neighborhood of $z$. In particular, any eventually constant net is convergent. A net converges in a product topology if and only if it converges coordinatewise; that is, $x_{\delta} \rightarrow z$ in $\prod_{j} Y_{j}$ if and only if $x_{\delta j} \rightarrow z_{j}$ in each $Y_{j}$.

\section{Main results}

Muranov's Lemma $\left({ }^{6}\right)$. Suppose that $\left(x_{\delta}\right)$ is a universal net in a set equipped with the cofinite topology. Then either $\left(x_{\delta}\right)$ converges to every point in the space, or $\left(x_{\delta}\right)$ is eventually constant.

Proof (without using AC or BPI). Suppose there is at least one point $z$ to which the net does not converge. Then $z$ has at least one open neighborhood $G$ for which we do not eventually have $x_{\delta} \in G$. Since the net is universal, eventually $x_{\delta} \in \complement G$, where $\complement$ denotes complement.

Now $G$ is nonempty (since it contains $z$ ), and it is an open set in a cofinite topology. Thus $\complement G$ is finite. Therefore $\left(x_{\delta}\right)$ is eventually constant.

Proof of $\mathrm{U} \Rightarrow \mathrm{TT}_{\mathrm{cf}}$. Let $\left\{Y_{j}: j \in J\right\}$ be a collection of topological spaces, each equipped with the cofinite topology. We are to show that the product topology on $P=\prod_{j \in J} Y_{j}$ is compact. Since the only topology on the empty set is a compact topology, we may assume that $P$ is nonempty. Thus we may assume that we are given some particular point $u \in P$; its $j$ th coordinate is some particular $u_{j} \in Y_{j}$.

Let $\left(x_{\delta}: \delta \in \mathbb{D}\right)$ be a universal net taking values in $P$. In view of principle $\mathrm{U}$, it suffices to show that $\left(x_{\delta}\right)$ has at least one limit in $P$. Since convergence of nets in product topologies is coordinatewise, it suffices to show that

$$
\prod_{j \in J}\left\{\text { limits of }\left(x_{\delta j}\right)\right\} \text { is nonempty, }
$$

$\left({ }^{5}\right)$ Strangely, although there are other universal nets besides the eventually constant ones, there are no other examples of universal nets; the existence arguments are all inherently nonconstructive. This makes universal nets difficult to visualize, which may be why many mathematicians are reluctant to use them. Nontrivial universal nets are a triumph of formalism: One might say that in this paper we are not really working with the universal nets themselves, but rather with sentences about hypothetical universal nets.

$\left({ }^{6}\right)$ I am grateful to Alexey Muranov, who extracted this lemma from an earlier version of my paper and thereby simplified things greatly. 
i.e., that we can choose a member of this product. But we may not use the Axiom of Choice, since we are trying to prove the equivalence of $U$ and $\mathrm{TT}_{\mathrm{cf}}$ as weakenings of AC. Thus, what we actually must show is how to nonarbitrarily choose a particular limit $z_{j}$ of the projected net $\left(x_{\delta j}\right)$ in each factor space $Y_{j}$.

We easily verify that $\left(x_{\delta j}\right)$ is universal in $Y_{j}$. Thus Muranov's Lemma is applicable. Now choose $z_{j}$ nonarbitrarily, by this rule:

- If $\left(x_{\delta j}\right)$ converges to every member of $Y_{j}$, then take $z_{j}=u_{j}$.

- Otherwise, $\left(x_{\delta j}\right)$ is eventually constant; let $z_{j}$ be the constant value that the net eventually assumes.

In either case, we have selected a particular $z_{j}$ for which $x_{\delta j} \rightarrow z_{j}$.

\section{References}

[1] E. Čech, On bicompact spaces, Ann. of Math. 38 (1937), 823-844.

[2] J. D. Halpern and A. Lévy, The Boolean prime ideal theorem does not imply the axiom of choice, in: Axiomatic Set Theory, Proc. Sympos. Pure Math. 13 (part 1), Amer. Math. Soc., Providence, RI, 1971, 83-134.

[3] P. Howard and J. E. Rubin, Consequences of the Axiom of Choice, Math. Surveys Monogr. 59, Amer. Math. Soc., Providence, RI, 1998.

[4] J. L. Kelley, The Tychonoff product theorem implies the axiom of choice, Fund. Math. 37 (1950), 75-76.

[5] - General Topology, Van Nostrand, New York, 1955; reprinted by Springer, New York, 1975.

[6] J. Łoś and C. Ryll-Nardzewski, On the application of Tychonoff's theorem in mathematical proofs, Fund. Math. 38 (1951), 233-237.

[7] J. Mycielski, Two remarks on Tychonoff's product theorem, Bull. Acad. Polon. Sci. Sér. Sci. Math. Astronom. Phys. 12 (1964), 439-441.

[8] F. Plastria, Two loose results in general topology, Bull. Soc. Math. Belg. 24 (1972), 380-385.

[9] E. Schechter, Handbook of Analysis and its Foundations, Academic Press, New York, 1996.

[10] A. Tychonoff, Über die topologische Erweiterung von Räumen, Math. Ann. 102 (1930), 544-561.

Department of Mathematics

Vanderbilt University

1326 Stevenson Center

Nashville, TN 37240, U.S.A.

E-mail: eric.schechter@vanderbilt.edu

Received 23 October 2005;

in revised form 3 December 2005 